\title{
Effect of body mass index and abdominal obesity on mortality after percutaneous coronary inter- vention: a nationwide, population-based study
}

\author{
Woo-Hyuk Song ${ }^{1,}$, Eun Hui Bae ${ }^{2,}$, Jeong Cheon $\mathrm{Ahn}^{1}$, Tae Ryom $\mathrm{Oh}^{2}$, Yong-Hyun Kim ${ }^{1}$, Jin Seok Kim ${ }^{1}$, \\ Sun-Won Kim ${ }^{1}$, Soo Wan Kim², Kyung-Do Han³ ${ }^{3}$ and Sang Yup Lim ${ }^{1}$
}

\begin{abstract}
${ }^{1}$ Department of Internal Medicine, Korea University Ansan Hospital, Ansan; ${ }^{2}$ Department of Internal Medicine, Chonnam National University Medical School, Gwangju; ${ }^{3}$ Department of Statistics and Actuarial Science, Soongsil University, Seoul, Korea
\end{abstract}

Received: March 17, 2020

Revised : April 3, 2020

Accepted: September 3, 2020

\section{Correspondence to}

Sang Yup Lim, M.D.

Department of Internal Medicine, Korea University

Ansan Hospital, 123 Jeokgeum-ro, Danwon-gu, Ansan 15355, Korea

Tel: $+82-31-8099-6352$

Fax: +82-31-412-6755

E-mail: vnlover@hanmail.net https://orcid.org/0000-00023042-6702

*These authors contributed equally to this work.
Background/Aims: We investigated the impact of obesity on the clinical outcomes following percutaneous coronary intervention (PCI).

Methods: We included South Koreans aged $>20$ years who underwent the Korean National Health Screening assessment between 2009 and 2012. Obesity was defined using the body mass index (BMI), according to the World Health Organization's recommendations. Abdominal obesity was defined using the waist circumference (WC), as defined by the Korean Society for Obesity. The odds and hazard ratios in all-cause mortality were calculated after adjustment for multiple covariates. Patients were followed up to the end of 2017.

Results: Among 130,490 subjects who underwent PCI, the mean age negatively correlated with BMI. WC, hypertension, diabetes, dyslipidemia, fasting glucose, total cholesterol, low-density lipoprotein cholesterol, and triglyceride levels correlated with the increased BMI. The mortality rates were higher in the lower BMI and WC groups than the higher BMI and WC groups. The non-obese with abdominal obesity group showed a mortality rate of 2.11 per 1,000 person-years. Obese with no abdominal obesity group had the lowest mortality rate (0.88 per 1,000 person-years). The mortality showed U-shaped curve with a cut-off value of 29 in case of BMI and $78 \mathrm{~cm}$ of WC.

Conclusions: The mortality showed U-shaped curve and the cut-off value of lowest mortality was 29 in case of BMI and $78 \mathrm{~cm}$ of WC. The abdominal obesity may be associated with poor prognosis in Korean patients who underwent PCI.

Keywords: Obesity; Coronary artery disease; Mortality

\section{INTRODUCTION}

The prevalence of obesity has been steadily and significantly increasing worldwide $[1,2]$. Because of its impact on cardiovascular diseases, obesity is becoming one of the most serious global health issues [3]. Obesity is strongly associated with the risk factors for atherosclerosis such as hypertension, diabetes mellitus (DM), dys- lipidemia, and can lead to atherosclerosis itself by increasing oxidative stress, inflammation, and endothelial dysfunction $[4,5]$. The risk factors associated with obesity and ischemic heart disease (IHD) are well-established, and obesity itself has been thought to be a risk factor of IHD and can worsen the prognosis regardless of metabolic status [6-9].

Previous studies showed that patients with obesity 
paradoxically exhibited more favorable clinical outcomes with respect to in-hospital, short-, and long-term mortality than those without obesity after percutaneous coronary intervention (PCI) [10-13]. These studies had defined obesity according to the body mass index (BMI), which has been the most frequently used measure of obesity. However, body adiposity differs according to age, sex, and ethnicity, and BMI alone is not able to distinguish between fatty and highly muscular individuals [14]. Waist circumference (WC) is another measure of obesity, which correlates well with abdominal fat distribution to discriminate visceral "unhealthy" obesity from "healthy" obesity $[15,16]$.

The purpose of this study was to address the relationship between obesity and long-term mortality in a large cohort of patients who underwent PCI. Furthermore, we included BMI and WC in the analysis of obesity, to determine which would be the better predictor of mortality.

\section{METHODS}

\section{The Korean National Health Insurance Service database}

The Korean National Health Insurance Service National Sample Cohort (NHIS-NSC) is constructed from the NHIS database and includes almost all citizens in Korea (50 million). The medical information recorded consists of patients' age, sex, living area, insurer payment coverage, deduction and claims data, and medical utilization/ transaction information. Within this cohort, 130,490 individuals with coronary artery disease treated by PCI between 2009 and 2012 were enrolled and followed-up for $5.57 \pm 0.29$ years until the end of 2017 (unless disqualified by death or emigration). Data regarding patient demographics and medical treatment claims for inpatient and outpatient care, including diagnoses, prescriptions, procedures, and nationwide health examination results, were reviewed. Diagnosis statements were defined by the International Classification of Diseases, tenth revision (ICD-10). Covariates and ICD-10 codes used in this study included DM (E11-14), hypertension (I10-13, I15), dyslipidemia (E78), IHD (I20), myocardial infarction (I21-22). We registered only de novo PCI and excluded patients with a history of PCI to avoid the effects of past coronary intervention, and those with cerebrovascular disease, heart failure, or cancer.

This study adhered to the tenets of the Declaration of Helsinki. As the database used in this study did not include personal identifiers, and this study was of retrospective observational nature, informed consent was waived and ethical approval was given by the Korea University Hospital Institutional Review Board (2019ASoo66).

\section{Anthropometric measurement and baseline charac- teristics}

Body weight $(\mathrm{kg})$ and height $(\mathrm{cm})$ were measured using an electronic scale, and WC (cm) was measured at the middle point between the rib cage and iliac crest by trained examiners. We defined obesity as a BMI $\geq 25 \mathrm{~kg} / \mathrm{m}^{2}$ and categorized patients into underweight (BMI $<18.5 \mathrm{~kg} / \mathrm{m}^{2}$ ), normal (BMI 18.5 to $23 \mathrm{~kg} / \mathrm{m}^{2}$ ), overweight (BMI 23 to 25 $\mathrm{kg} / \mathrm{m}^{2}$ ), stage I obesity (BMI 25 to $30 \mathrm{~kg} / \mathrm{m}^{2}$ ), and stage 2 obesity $\left(\mathrm{BMI} \geq 30 \mathrm{~kg} / \mathrm{m}^{2}\right)$ according to the World Health Organization recommendations for Asians [17]. Abdominal obesity was defined as WC $\geq 90 \mathrm{~cm}$ in men and 85 $\mathrm{cm}$ in women according to the definition of the Korean Society for the Study of Obesity [18]. WC was divided into six categories with increments of $5 \mathrm{~cm}$ : level $1,<80 \mathrm{~cm}$ in men and $<75 \mathrm{~cm}$ in women; level 2,80 to $85 \mathrm{~cm}$ in men and 75 to $80 \mathrm{~cm}$ in women; level 3,85 to $90 \mathrm{~cm}$ in men and 80 to $85 \mathrm{~cm}$ in women; level 4,90 to $95 \mathrm{~cm}$ in men and 85 to $90 \mathrm{~cm}$ in women; level 5,95 to $100 \mathrm{~cm}$ in men and 90 to $95 \mathrm{~cm}$ in women; and level $6, \geq 100 \mathrm{~cm}$ in men and $\geq 95$ $\mathrm{cm}$ in women.

Baseline characteristics and health-related behaviors such as income, smoking, alcohol consumption, and exercise were confirmed through standardized questionnaires. All blood samples were collected after fasting, and blood pressure was measured using a sphygmomanometer after 15 minutes of rest. The diagnosis of diabetes, hypertension, and hyperlipidemia was confirmed using laboratory data (fasting blood glucose level $\geq 126$ $\mathrm{mg} / \mathrm{dL}$; systolic blood pressure $\geq 140 \mathrm{mmHg}$ and diastolic blood pressure $\geq 90 \mathrm{mmHg}$; and total cholesterol levels $\geq 240 \mathrm{mg} / \mathrm{dL}$, respectively) or ICD code (ICD-10 codes E11-14, I10-I15, or E78, respectively) with a claim of for medication specific to the individual disease. 


\section{General health-related behavior and socio-demo- graphic variables}

Patients were also categorized according to smoking status (non-smokers, former smokers, or current smoker) and alcohol consumption (none, moderate, or heavy drinkers [ $\geq 3$ days/week]). Regular exercise was defined as vigorous physical activity for at least $20 \mathrm{~min} /$ day. Residence in urban areas was also checked and income was divided according to quartiles, with Q1 being the lowest and $\mathrm{Q}_{4}$ the highest.

\section{Outcomes}

All-cause mortality was checked between 2010 and 2017 for each participant, and the number of person-years of follow-up was also recorded. All-cause mortality was assessed within $5.57 \pm 0.29$ years after the last recorded WC value and there were a total of 10,560 deaths.

\section{Statistical analysis}

The general characteristics of the patients are presented as mean \pm standard deviation (SD) for continuous variables and percentages $(\mathrm{SD})$ for categorical variables. Analysis of variance (ANOVA) was used to compare continuous variables, and a chi-square test was used to compare categorical variable. The hazard ratios (HRs) and $95 \%$ confidence intervals (CIs) for all-cause mortality were analyzed using multivariable Cox proportional hazard models, after adjusting for age and sex in model 1; and age, sex, smoking, alcohol consumption, regular physical activity, low-income status, hypertension, diabetes, dyslipidemia, and chronic kidney disease in model 2 . The HR and 95\% CI for all-cause mortality according to the six WC groups were also calculated for the different subgroups according to sex and age (20-40, 40-64, and 65-84 years) using a multivariable Cox model after adjusting for all covariates. We also calculated the HRs and 95\% CI for all-cause mortality with respect to WC, with group 3 being the reference, across the five BMI groups. All statistical analyses were performed using SAS version 9.3 (SAS Institute Inc., Cary, NC, USA), and $p<0.05$ for two-tailed $t$ tests was considered statistically significant.

\section{RESULTS}

\section{Baseline characteristics of the study population}

Table 1 shows the baseline characteristics of the participants according to the BMI. Hypertension, diabetes, dyslipidemia, fasting glucose level, total cholesterol, low-density lipoprotein cholesterol, and triglyceride levels were positively correlated and the mean age was reversely correlated with BMI. In addition, the WC also increased in parallel with the BMI. The proportion of non-smokers, non-alcohol drinkers, and the lowest income level (Q1) showed a U-shaped curve.

\section{BMI, WC, and mortality}

The highest mortality was seen in the underweight (BMI $\left.<18.5 \mathrm{~kg} / \mathrm{m}^{2}\right)$ group, and stage 2 obesity $\left(\mathrm{BMI} \geq 30 \mathrm{~kg} / \mathrm{m}^{2}\right)$ was associated with the lowest mortality rates in the multivariate analysis (Table 2). The association between BMI and mortality showed an inverse correlation up to a cutoff of $29 \mathrm{~kg} / \mathrm{m}^{2}$ after adjusting for WC in model 3 (Fig. $1 \mathrm{~A}$ ).

The lowest WC group ( $<80 \mathrm{~cm}$ in men and $<75 \mathrm{~cm}$ in women) showed the highest mortality rates. However, the lowest mortality rates were observed in 90 to $95 \mathrm{~cm}$ in men and 85 to $90 \mathrm{~cm}$ in women (Table 2). The association between WC and mortality showed a J-shaped curve after adjusting for BMI in model $_{3}$ (Fig. 1B).

\section{Subgroup analyses}

Subgroup analysis of the relationship between obesity and mortality was performed according to BMI or WC subgroups (Fig. 2). After adjusting for all covariates, every subgroup with obesity according to BMI or abdominal obesity according to WC showed lower mortality than those without obesity or abdominal obesity.

Table 3 shows the mortality rate according to obesity defined by BMI or WC. The highest mortality was observed in the non-obese with abdominal obesity group (2.11 per 1,000 person-years) while the lowest was in the obese with no abdominal obesity group ( 0.88 per 1,000 person-years). Analysis of the HRs according to BMI showed a reverse correlation with mortality until a cutoff of $29.09 \mathrm{~kg} / \mathrm{m}^{2}$, where the lowest mortality risk was observed. In terms of WC, $78 \mathrm{~cm}$ was the point at which the lowest HR was observed (Fig. 1). 
Table 1. General characteristics of participants by body mass index $\left(\mathrm{kg} / \mathrm{m}^{2}\right)$

\begin{tabular}{|c|c|c|c|c|c|c|}
\hline \multirow{2}{*}{ Characteristic } & \multicolumn{5}{|c|}{ BMI, kg/m ${ }^{2}$} & \multirow{2}{*}{$p$ value } \\
\hline & $<18.5$ & $18.5-23$ & $23-25$ & $25-30$ & $>30$ & \\
\hline Number & 1,860 & 34,248 & 36,345 & 52,012 & 6,025 & \\
\hline Age, yr & $70.66 \pm 10.01$ & $66.01 \pm 10.09$ & $63.89 \pm 9.82$ & $62.15 \pm 10.11$ & $59.07 \pm 11.49$ & $<0.0001$ \\
\hline$<65$ & $459(24.68)$ & $14,593(42.61)$ & $18,614(51.21)$ & $29,948(57.58)$ & $3,926(65.16)$ & $<0.0001$ \\
\hline$\geq 65$ & $1,401(75 \cdot 32)$ & $19,655(57 \cdot 39)$ & $17,731(48.79)$ & $22,064(42.42)$ & $2,099(34.84)$ & \\
\hline Male sex & $1,231(66.18)$ & $24,099(70.37)$ & $26,992(74.27)$ & $38,387(73.8)$ & $3,875(64 \cdot 32)$ & $<0.0001$ \\
\hline Place (urban) & $713(38.42)$ & $14,373(42.01)$ & $15,762(43.4)$ & $22,158(42.63)$ & $2,500(41.51)$ & $<0.0001$ \\
\hline $\mathrm{BMI}, \mathrm{kg} / \mathrm{m}^{2}$ & $17.48 \pm 0.89$ & $21.49 \pm 1.13$ & $24.03 \pm 0.57$ & $26.83 \pm 1.29$ & $31.85 \pm 1.85$ & $<0.0001$ \\
\hline Height, cm & $159.83 \pm 9.16$ & $161.92 \pm 8.81$ & $162.94 \pm 8.57$ & $163.17 \pm 8.84$ & $162.52 \pm 9.94$ & $<0.0001$ \\
\hline Weight, kg & $44.81 \pm 5.61$ & $56.52 \pm 6.92$ & $63.98 \pm 6.82$ & $71.64 \pm 8.38$ & $84.4 \pm 11.1$ & $<0.0001$ \\
\hline WC, cm & $70.37 \pm 6.28$ & $78.83 \pm 5.8$ & $84.24 \pm 5.21$ & $89.78 \pm 5.88$ & $99.11 \pm 6.99$ & $<0.0001$ \\
\hline $\mathrm{SBP}, \mathrm{mm} \mathrm{Hg}$ & $122.89 \pm 17.54$ & $124.44 \pm 16.27$ & $125.82 \pm 15.55$ & $127.71 \pm 15.36$ & $130.85 \pm 15.52$ & $<0.0001$ \\
\hline DBP, mm Hg & $74.24 \pm 10.31$ & $74.91 \pm 10.06$ & $75.99 \pm 9.88$ & $77.42 \pm 9.9$ & $79.77 \pm 10.14$ & $<0.0001$ \\
\hline Smoke & & & & & & $<0.0001$ \\
\hline Non & $995(53.49)$ & $17,548(51.24)$ & $17,616(48.47)$ & $24,473(47.05)$ & $2,939(48.78)$ & \\
\hline Ex & $470(25.27)$ & $10,917(31.88)$ & $13,170(36.24)$ & $19,665(37.81)$ & $2,058(34.16)$ & \\
\hline Current & $395(21.24)$ & 5,783 (16.89) & $5,559(15 \cdot 3)$ & $7,874(15.14)$ & $1,028(17.06)$ & \\
\hline Drink & & & & & & $<0.0001$ \\
\hline Non & $1,542(82.9)$ & $25,438(74.28)$ & $25,589(70.41)$ & $35,857(68.94)$ & $4,209(69.86)$ & \\
\hline Mild & $283(15.22)$ & $7,981(23 \cdot 3)$ & $9,775(26.9)$ & $14,449(27.78)$ & $1,568(26.02)$ & \\
\hline Heavy & $35(1.88)$ & $829(2.42)$ & $981(2.7)$ & $1,706(3.28)$ & $248(4.12)$ & \\
\hline Regular exercise & $586(31.51)$ & $15,247(44 \cdot 52)$ & $17,993(49 \cdot 51)$ & $25,810(49.62)$ & $2,709(44.96)$ & $<0.0001$ \\
\hline Income (low) & $501(26.94)$ & $8,588(25.08)$ & $8,764(24.11)$ & $12,609(24.24)$ & $1,524(25.29)$ & 0.0008 \\
\hline Diabetes & $521(28.01)$ & $11,307(33.02)$ & $12,551(34 \cdot 53)$ & $18,882(36.3)$ & $2,736(45 \cdot 41)$ & $<0.0001$ \\
\hline Hypertension & $1,333(71.67)$ & $25,929(75.71)$ & $28,960(79.68)$ & $43,684(83.99)$ & $5,369(89.11)$ & $<0.0001$ \\
\hline Dyslipidemia & $1,483(79 \cdot 73)$ & $29,867(87.21)$ & $32,462(89.32)$ & $47,140(90.63)$ & $5,490(91.12)$ & $<0.0001$ \\
\hline CKD & $388(20.86)$ & $5,861(17.11)$ & $5,759(15.85)$ & $8,320(16)$ & $1,064(17.66)$ & $<0.0001$ \\
\hline FBS, mg/dL & $106.01 \pm 36.27$ & $108.19 \pm 33.22$ & $109.96 \pm 32.92$ & $111.37 \pm 32.16$ & $116.6 \pm 35.47$ & $<0.0001$ \\
\hline $\mathrm{TC}, \mathrm{mg} / \mathrm{dL}$ & $152.83 \pm 34.31$ & $151.14 \pm 34.65$ & $152.25 \pm 34.89$ & $154.14 \pm 35.21$ & $158.92 \pm 36.67$ & $<0.0001$ \\
\hline HDL-C, mg/dL & $53.1 \pm 13.86$ & $49.81 \pm 14.14$ & $47.85 \pm 14.37$ & $46.62 \pm 14.09$ & $46.24 \pm 15 \cdot 5$ & $<0.0001$ \\
\hline LDL-C, mg/dL & $79.86 \pm 29.44$ & $78.43 \pm 29.85$ & $78.81 \pm 29.98$ & $79.18 \pm 30.25$ & $80.87 \pm 31.41$ & $<0.0001$ \\
\hline $\mathrm{TG}, \mathrm{mg} / \mathrm{dL}$ & $\begin{array}{c}91.1 \\
(89.26-92.98)\end{array}$ & $\begin{array}{c}103.27 \\
(102.75-103.8)\end{array}$ & $\begin{array}{c}116.42 \\
(115.83-117.01)\end{array}$ & $\begin{array}{c}129.14 \\
(128.59-129.68)\end{array}$ & $\begin{array}{c}147.47 \\
(145.63-149.33)\end{array}$ & $<0.0001$ \\
\hline
\end{tabular}

Values are presented as mean \pm standard deviation, number (\%), or median (range). $p<0.0001$ for all data.

BMI, body mass index; WC, waist circumference; SBP, systolic blood pressure; DBP, diastolic blood pressure; CKD, chronic kidney disease; FBS, fasting blood sugar; TC, total cholesterol; TG, triglyceride; HDL-C, high density lipoprotein cholesterol; LDL-C, low density lipoprotein cholesterol.

\section{DISCUSSION}

We presented the findings of a large-scale cohort study assessing the relationship between obesity by BMI or abdominal obesity by WC and mortality in patients with coronary artery disease who underwent PCI. This na- 
Table 2. Mortality rate and multivariate-adjusted HR (95\% CI) according to baseline BMI and WC

\begin{tabular}{|c|c|c|c|c|c|c|c|}
\hline Variable & $\begin{array}{l}\text { Total } \\
\text { no. }\end{array}$ & $\begin{array}{l}\text { No. of } \\
\text { events }\end{array}$ & $\begin{array}{l}\text { Follow-up } \\
\text { duration, } \\
\text { day }\end{array}$ & $\begin{array}{l}\text { Mortality } \\
\text { rate, /1,০oo } \\
\text { person-years }\end{array}$ & Model 1 & Model 2 & Model 3 \\
\hline \multicolumn{8}{|l|}{$\mathrm{BMI}, \mathrm{kg} / \mathrm{m}^{2}$} \\
\hline$<18.5$ & 1,860 & 504 & $8,210.96$ & 6.13814 & $1.881(1.714-2.064)$ & $1.85(1.685-2.03)$ & $1.818(1.65-2.003)$ \\
\hline $18.5-23$ & 34,248 & 4,068 & $169,235.58$ & 2.40375 & 1 (reference) & 1 (reference) & 1 (reference) \\
\hline $23-25$ & 36,345 & 2,621 & $184,363.13$ & 1.42165 & $0.697(0.664-0.732)$ & $0.698(0.665-0.734)$ & $0.687(0.65-0.725)$ \\
\hline $25-30$ & 52,012 & 3,043 & $265,937.48$ & 1.14425 & $0.645(0.615-0.676)$ & $0.637(0.607-0.668)$ & $0.584(0.548-0.622)$ \\
\hline$>30$ & 6,025 & 324 & $29,873.79$ & 1.08456 & $0.793(0.708-0.889)$ & $0.725(0.646-0.813)$ & $0.57(0.497-0.655)$ \\
\hline \multicolumn{8}{|c|}{ WC, cm (male/female) } \\
\hline$<80 / 75$ & 20,799 & 2,311 & $102,048.49$ & 2.26461 & $1.402(1.325-1.484)$ & $1.435(1.356-1.519)$ & $1.017(0.952-1.086)$ \\
\hline $85 / 80$ & 29,006 & 2,249 & $146,269.48$ & 1.53757 & $1.062(1.003-1.124)$ & $1.084(1.024-1.147)$ & $0.94(0.886-0.997)$ \\
\hline $90 / 85$ & 34,457 & 2,513 & $175,117 \cdot 38$ & 1.43504 & 1 (reference) & 1 (reference) & 1 (reference) \\
\hline $95 / 90$ & 25,766 & 1,870 & $131,001.52$ & 1.42746 & $0.966(0.91-1.025)$ & $0.947(0.892-1.006)$ & $1.064(1-1.132)$ \\
\hline $100 / 95$ & 12,864 & 941 & $65,335 \cdot 25$ & 1.44026 & $0.974(0.904-1.05)$ & $0.915(0.849-0.986)$ & $1.099(1.014-1.192)$ \\
\hline$>100 / 95$ & 7,598 & 676 & $37,848.81$ & 1.78605 & $1.232(1.131-1.341)$ & $1.1(1.01-1.198)$ & $1.363(1.233-1.506)$ \\
\hline
\end{tabular}

p 0.0001 for all data. Model 1 was adjusted for age and sex. Model 2: model $1+$ smoking, alcohol drinking, regular physical activity, low-income status, hypertension, diabetes, dyslipidemia and chronic kidney disease. Model 3: Model $2+$ WC (BMI group) or BMI (WC group).

HR, hazard ratio; CI, confidence interval; BMI, body mass index; WC, waist circumference.

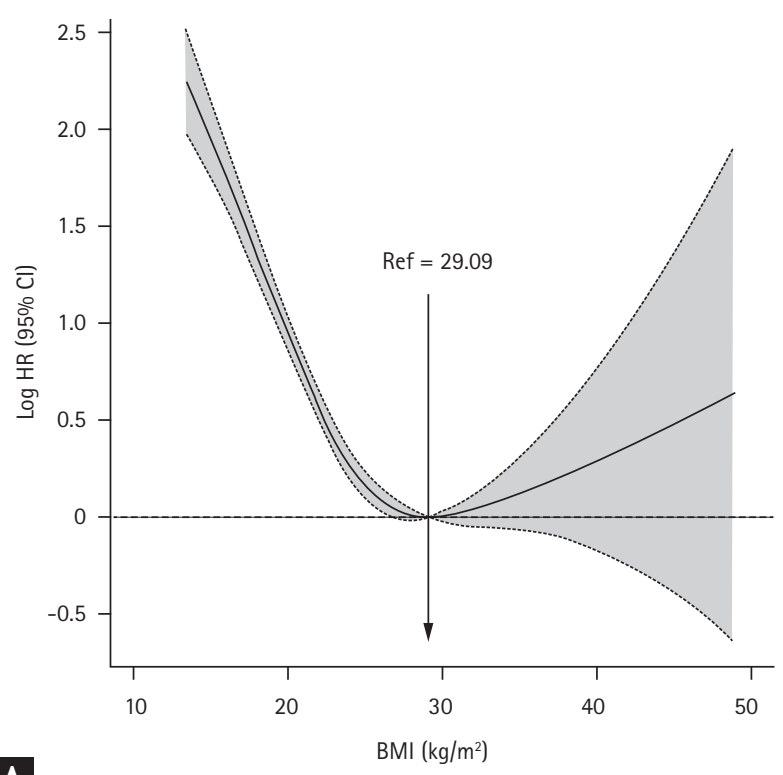

A

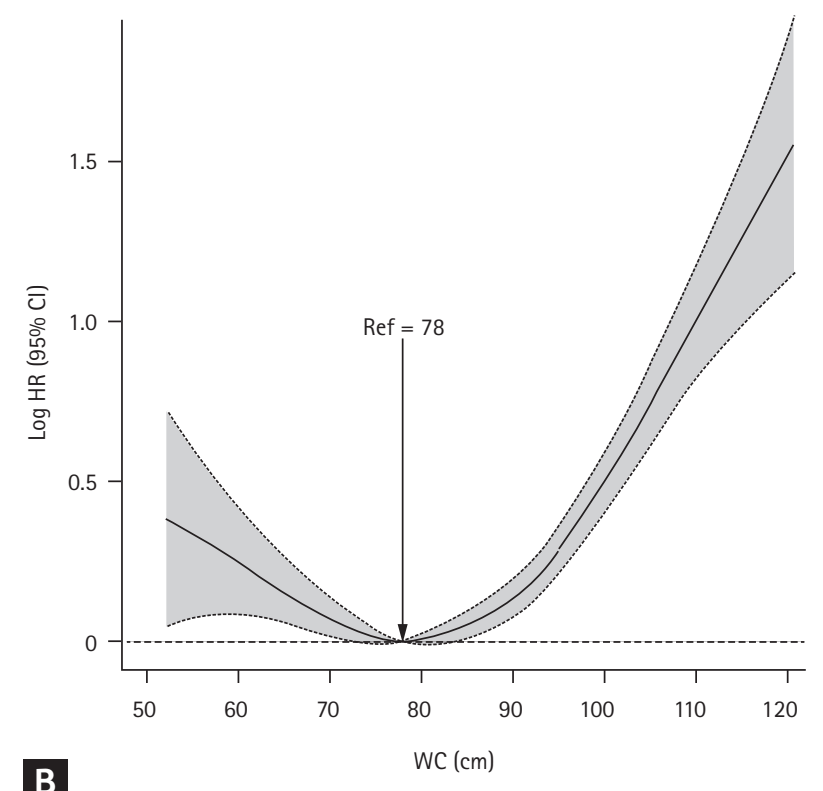

B

Figure 1. Hazard ratio (HR) for all-cause mortality. (A) Body mass index (BMI): Adjusted for age, sex, smoking, drinking, exercise, income, diabetes, dyslipidemia, hypertension, and chronic kidney disease. (B) Waist circumference (WC): Adjusted for age, sex, smoking, drinking, exercise, income, diabetes, dyslipidemia, hypertension and chronic kidney disease. CI, confidence interval. 

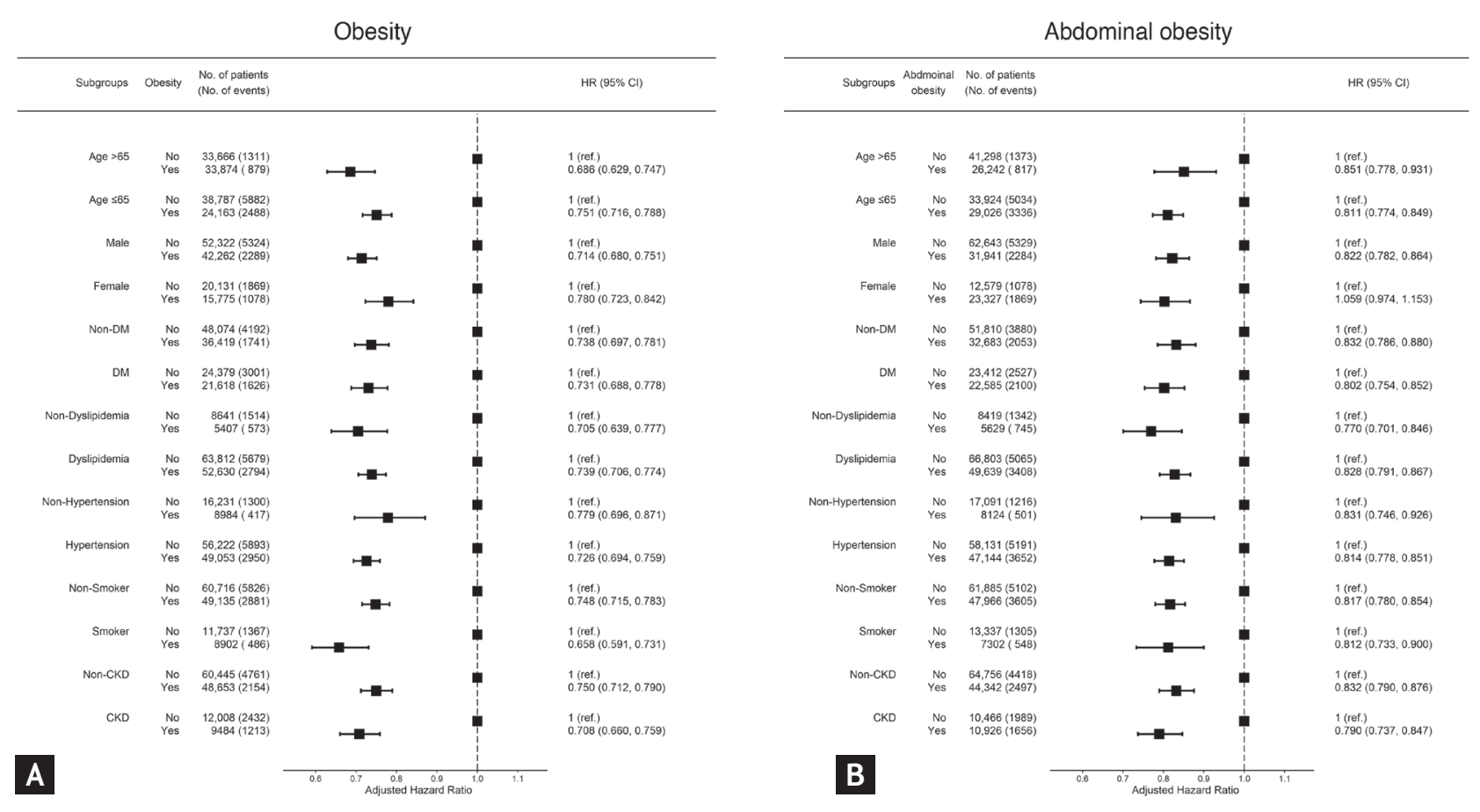

Figure 2. Subgroup analysis for obesity (A) and abdominal obesity (B). HR, hazard ratio; CI, confidence interval; DM, diabetes mellitus; CKD, chronic kidney disease.

Table 3. The mortality rate (per 1,00o person years) according to obesity by BMI and abdominal obesity by WC

\begin{tabular}{lccccc}
\hline Obesity & Abdominal obesity & $\begin{array}{c}\text { No. of } \\
\text { patients }\end{array}$ & $\begin{array}{c}\text { No. of } \\
\text { events }\end{array}$ & $\begin{array}{c}\text { Follow-up } \\
\text { duration, day }\end{array}$ & $\begin{array}{c}\text { Mortality rate, } \\
\text { /1,00o person-years }\end{array}$ \\
\hline No & No & 57,898 & 5,635 & $287,877.81$ & 1.95743 \\
& Yes & 14,555 & 1,558 & $73,931.85$ & 2.10735 \\
Yes & No & 17,324 & 772 & $88,123.88$ & 0.87604 \\
& Yes & 40,713 & 2,595 & $207,687.4$ & 1.24947 \\
\hline
\end{tabular}

p $<0.0001$ for all data. Multivariate-adjusted hazard ratio (95\% confidence interval) by age, sex, BMI, smoking, prescence of diabetes, dyslipidemia, hypertension, and incidence rate.

BMI, body mass index; WC, waist circumference.

tionwide study included nearly half of the adult population of South Korea. To our knowledge, this is one of the largest studies of its type to be conducted on a large, homogenous nationwide population-based cohort.

In our study, the mortality after PCI showed the U shaped-curve, the cut-off value of lowest mortality was 29 in case of BMI and $78 \mathrm{~cm}$ of WC and increased mortality was observed at the non-obese with abdominal obesity group. In the analysis of the BMI model, the mortality was highest in the lowest BMI group (BMI < $18.5 \mathrm{~kg} / \mathrm{m}^{2}$ ) and lowest in the highest BMI group (BMI > $30 \mathrm{~kg} / \mathrm{m}^{2}$ ), and showed a U-shaped pattern. After adjust- ment for WC, the mortality in the overweight to obese BMI group was relatively attenuated and the mortality of the underweight group further increased. In addition, these results showed the importance of abdominal obesity on mortality after PCI. The non-obese with abdominal obesity group had a higher risk of mortality than the other groups and the obese with no abdominal obesity group showed the lowest mortality. Abdominal obesity acted as a poor prognostic factor among both obese and non-obese group. The underweight group with abdominal obesity (i.e., the lean but metabolically unhealthy group) showed a worse prognosis after PCI than the 
obese group. Therefore, abdominal obesity may be associated with poor prognosis in patients with coronary artery disease who undergo PCI.

Individuals with abdominal obesity were found to have a higher risk of atherosclerosis and subclinical cardiovascular disease [19-22]. The Metabolic Efficiency With Ranolazine for Less Ischemia in non-ST elevation acute coronary syndrome-Thrombolysis in Myocardial Infarction (MERLIN-TIMI-36) study, a study on the relationship between abdominal obesity and cardiovascular outcomes in 6,560 patients with acute coronary syndrome, WC greater than proportionate to BMI were at the highest risk of developing cardiovascular events [23]. Meta-analysis of 15,923 patients with CAD showed that even non-obsese with abdominal obesity is associated with the highest risk of mortality and waist-to-hip ratio is more precise predictor of poor prognosis than BMI [24]. Park et al. [25] demonstrated that abdominal obesity with truncal fat is associated with unfavorable clinical outcomes after drug eluting stent implantation and is more clinically relevant than BMI in Korean patients. The major pathogenic drivers associated with poor prognosis of abdominal obesity after PCI is that the visceral fat promotes coronary artery disease progression such as progression of atherosclerosis, increasing coronary plaque vulnerability that causes acute coronary syndrome via increased inflammatory cytokines, oxidative stress, insulin resistance and systemic inflammation, etc. [26,27].

The reason of higher mortality in the underweight group than the normal BMI or obese groups might be due to overall health of underweight people is inevitably more vulnerable than normal or obese people. The underweight group may be related to an imbalance in nutrition, which lead to developing cardiovascular or cerebrovascular and inflammatory diseases. Although all eligible patients were prescribed medications in accordance with guideline-based treatment, patients in the lower BMI group might have received optimal medical therapy, including aspirin, newer $\mathrm{P}_{2} \mathrm{Y}_{12}$ inhibitors, renin-angiotensin-aldosterone system blockers, and statins, at lower rates due to various reasons such as bleeding, malnutrition, or reduced blood pressure $[28,29]$. In addition, the lower BMI group clearly exhibited adverse drug effects despite receiving the usual dosage, and the success rate of PCI did not differ compared with the obese groups. In a meta-analysis of sex-specific relationships between BMI and coronary heart disease, a higher risk was observed in underweight patients of both sexes than in those with normal weight [30].

Our study has several limitations. First, the prognosis was observed through BMI and WC only at the point of PCI, and there is no follow-up data to confirm the changes in BMI and WC. Second, there are conflicting definitions of abdominal obesity in the literature. Although we adopted the definition of abdominal obesity, one of the components of metabolic syndrome that was modified for our South Korean population, the widely-used criteria for each metabolic syndrome component disregard ethnic and regional differences. Third, the definition of obesity according to BMI is still debated. Although lean mass index, assessments of body fat, or fat-free mass might be expected to be accurate measures of true body fat levels, we adopted the BMI because it is simple, universal, and reproducible. Fourth, our data could not reflect the effect of exercise or diet on weight reduction during the follow-up period. So, detailed data on medication (e.g., brand name, dose, and duration) and follow-up laboratory findings were not fully available. Finally, it was difficult to determine from the NHIC database whether the patients' medications following PCI were prescribed according to guideline-based treatment protocols. To overcome these limitations, we plan to expand on our research and will continue to collect relevant data.

In conclusion, the impact of obesity on the clinical outcomes in Korean patient who underwent PCI showed U-shaped curve on the mortality, and a cut-off value of lowest mortality was 29 in case of BMI and $78 \mathrm{~cm}$ in case of WC. In addition, the presence of abdominal obesity was associated with an unfavorable prognosis after PCI.

\section{KEY MESSAGE}

1. We investigated the impact of obesity on the clinical outcomes following percutaneous coronary intervention (PCI).

2. The mortality showed U-shaped curve, with a cut-off value of 29 in case of body mass index and $78 \mathrm{~cm}$ in case of waist circumference after PCI.

3. The presence of abdominal obesity was associated with an unfavorable prognosis after PCI. 


\section{Conflict of interest}

No potential conflict of interest relevant to this article was reported.

\section{Acknowledgments}

This research was supported by the Bio \& Medical Development Program of the National Research Foundation (NRF) funded by the Korean government (MSIT) (2017M3A9E8023001), grant of the Korea Health Technology R\&D Project through the Korea Health Industry Development Institute (KHIDI), funded by the Ministry of Health \& Welfare, Republic of Korea (grant number: HI18Co331), Korea University Ansan Hospital (K1923581) and by a grant (BCRI 18021\&20025) of Chonnam National University Hospital Biomedical Research Institute.

\section{REFERENCES}

1. Yoon KH, Lee JH, Kim JW, et al. Epidemic obesity and type 2 diabetes in Asia. Lancet 2006;368:1681-1688.

2. Patterson RE, Frank LL, Kristal AR, White E. A comprehensive examination of health conditions associated with obesity in older adults. Am J Prev Med 2004;27:385-390.

3. An R, Ji M, Zhang S. Global warming and obesity: a systematic review. Obes Rev 2018;19:150-163.

4. Alexander CM, Landsman PB, Teutsch SM, Haffner SM; Third National Health and Nutrition Examination Survey (NHANES III); National Cholesterol Education Program (NCEP). NCEP-defined metabolic syndrome, diabetes, and prevalence of coronary heart disease among NHANES III participants age 50 years and older. Diabetes 2003;52:12101214.

5. Bays HE, Gonzalez-Campoy JM, Bray GA, et al. Pathogenic potential of adipose tissue and metabolic consequences of adipocyte hypertrophy and increased visceral adiposity. Expert Rev Cardiovasc Ther 2008;6:343-368.

6. Prospective Studies Collaboration, Whitlock G, Lewington $\mathrm{S}$, et al. Body-mass index and cause-specific mortality in 900 ooo adults: collaborative analyses of 57 prospective studies. Lancet 2009;373:1083-1096.

7. Manson JE, Colditz GA, Stampfer MJ, et al. A prospective study of obesity and risk of coronary heart disease in women. N Engl J Med 1990;322:882-889.

8. Nordestgaard BG, Palmer TM, Benn M, et al. The effect of elevated body mass index on ischemic heart disease risk: causal estimates from a Mendelian randomisation approach. PLoS Med 2012;9:e1001212.

9. Ramachandran A, Chamukuttan S, Shetty SA, Arun N, Susairaj P. Obesity in Asia: is it different from rest of the world. Diabetes Metab Res Rev 2012;28 Suppl 2:47-51.

10. Ellis SG, Elliott J, Horrigan M, Raymond RE, Howell G. Low-normal or excessive body mass index: newly identified and powerful risk factors for death and other complications with percutaneous coronary intervention. Am J Cardiol 1996;78:642-646.

11. Gruberg L, Weissman NJ, Waksman R, et al. The impact of obesity on the short-term and long-term outcomes after percutaneous coronary intervention: the obesity paradox? J Am Coll Cardiol 2002;39:578-584.

12. Dhoot J, Tariq S, Erande A, Amin A, Patel P, Malik S. Effect of morbid obesity on in-hospital mortality and coronary revascularization outcomes after acute myocardial infarction in the United States. Am J Cardiol 2013;111:1104-1110.

13. Oreopoulos A, Padwal R, Norris CM, Mullen JC, Pretorius V, Kalantar-Zadeh K. Effect of obesity on short- and longterm mortality postcoronary revascularization: a meta-analysis. Obesity (Silver Spring) 2008;16:442-450.

14. Mathieu P, Pibarot P, Larose E, Poirier P, Marette A, Despres JP. Visceral obesity and the heart. Int J Biochem Cell Biol 2008;40:821-836.

15. Browning LM, Hsieh SD, Ashwell M. A systematic review of waist-to-height ratio as a screening tool for the prediction of cardiovascular disease and diabetes: 0.5 could be a suitable global boundary value. Nutr Res Rev 2010;23:247269.

16. World Health Organization. Waist Circumference and Waist-Hip Ratio: Report of a WHO Expert Consultation. Geneva (CH): World Health Organization, 2011.

17. World Health Organization. The Asia-Pacific Perspective: Redefining Obesity and Its Treatment. Sydney (AU): Health Communications Australia, 2000.

18. Yoon YS, Oh SW. Optimal waist circumference cutoff values for the diagnosis of abdominal obesity in Korean adults. Endocrinol Metab (Seoul) 2014;29:418-426.

19. Kim S, Kyung C, Park JS, et al. Normal-weight obesity is associated with increased risk of subclinical atherosclerosis. Cardiovasc Diabetol 2015;14:58.

20. Ryo M, Kishida K, Nakamura T, Yoshizumi T, Funahashi T, Shimomura I. Clinical significance of visceral adiposity assessed by computed tomography: a Japanese perspective. World J Radiol 2014;6:409-416. 
21. Kassi E, Pervanidou P, Kaltsas G, Chrousos G. Metabolic syndrome: definitions and controversies. BMC Med 2011;9:48.

22. Lim S, Meigs JB. Ectopic fat and cardiometabolic and vascular risk. Int J Cardiol 2013;169:166-176.

23. Kadakia MB, Fox CS, Scirica BM, Murphy SA, Bonaca MP, Morrow DA. Central obesity and cardiovascular outcomes in patients with acute coronary syndrome: observations from the MERLIN-TIMI 36 trial. Heart 2011;97:1782-1787.

24. Coutinho T, Goel K, Correa de Sa D, et al. Combining body mass index with measures of central obesity in the assessment of mortality in subjects with coronary disease: role of "normal weight central obesity". J Am Coll Cardiol 2013;61:553-560.

25. Park SJ, Lim HS, Sheen SS, et al. Impact of body fat distribution on long-term clinical outcomes after drug-eluting stent implantation. PLoS One 2018;13:eo197991.

26. Alexopoulos N, Katritsis D, Raggi P. Visceral adipose tissue as a source of inflammation and promoter of atherosclerosis. Atherosclerosis 2014;233:104-112.

27. Ragino YI, Stakhneva EM, Polonskaya YV, Kashtanova EV.
The role of secretory activity molecules of visceral adipocytes in abdominal obesity in the development of cardiovascular disease: a review. Biomolecules 2020;10:374.

28. Numasawa Y, Kohsaka S, Miyata H, et al. Impact of body mass index on in-hospital complications in patients undergoing percutaneous coronary intervention in a Japanese real-world multicenter registry. PLoS One 2015;10:e0124399.

29. Azhari Z, Ismail MD, Zuhdi ASM, Md Sari N, Zainal Abidin I, Wan Ahmad WA. Association between body mass index and outcomes after percutaneous coronary intervention in multiethnic South East Asian population: a retrospective analysis of the Malaysian National Cardiovascular Disease Database-Percutaneous Coronary Intervention (NCVD-PCI) registry. BMJ Open 2017;7:eo17794.

30. Mongraw-Chaffin ML, Peters SAE, Huxley RR, Woodward M. The sex-specific association between BMI and coronary heart disease: a systematic review and meta-analysis of 95 cohorts with 1.2 million participants. Lancet Diabetes Endocrinol 2015;3:437-449. 